\title{
Neuroblastoma cells injected into experimental mature teratoma reveal a tropism for embryonic loose mesenchyme
}

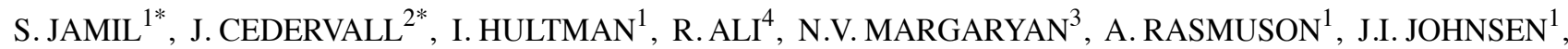 \\ B. SVEINBJÖRNSSON ${ }^{5}$, T. DALIANIS ${ }^{6}$, L. KANTER ${ }^{6}$, A. ORREGO ${ }^{6}$, L. STRIZZI ${ }^{3}$, M.J.C. HENDRIX ${ }^{3}$, \\ B. SANDSTEDT ${ }^{1}$, P. KOGNER ${ }^{1}$ and L. ÄHRLUND-RICHTER ${ }^{1}$ \\ ${ }^{1}$ Department of Women's and Children's Health, Karolinska Institute, Stockholm; \\ ${ }^{2}$ Department of Medical Biochemistry and Microbiology, Uppsala University, Uppsala, Sweden; \\ ${ }^{3}$ Ann \& Robert H. Lurie Children's Hospital of Chicago Research Center, Robert H. Lurie Comprehensive \\ Cancer Center, Chicago, IL, USA; ${ }^{4}$ Department of Neuroscience, Karolinska Institute, Stockholm, \\ Sweden; ${ }^{5}$ Division of Molecular Inflammation Research, University of Tromsö, Tromsö, Norway; \\ ${ }^{6}$ Department of Oncology and Pathology, Karolinska Institute, Stockholm, Sweden
}

Received May 14, 2013; Accepted June 26, 2013

DOI: 10.3892/ijo.2013.2014

\begin{abstract}
Embryonic neural tumors are responsible for a disproportionate number of cancer deaths in children. Although dramatic improvements in survival for pediatric malignancy has been achieved in previous years advancements seem to be slowing down. For the development of new enhanced therapy and an increased understanding of the disease, pre-clinical models better capturing the neoplastic niche are essential. Tumors of early childhood present in this respect a particular challenge. Here, we explore how components of the embryonic process in stem-cell induced mature teratoma can function as an experimental in vivo microenvironment instigating the growth of injected childhood neuroblastoma (NB) cell lines. Three human NB cell lines, IMR-32, Kelly and SK-N-BE(2), were injected into mature pluripotent stem cell-induced teratoma (PSCT) and compared to xenografts of the same cell lines. Proliferative NB cells from all lines were readily detected in both models with a typical histology of a poorly differentiated NB tumor with a variable amount of fibrovascular stroma. Uniquely in the PSCT microenvironment, NB cells were found integrated in a non-random fashion. Neuroblastoma cells were never observed in areas with well-differentiated somatic tissue i.e. bone, muscle, gut or areas of other easily identifiable tissue types. Instead, the three cell lines all showed initial growth exclusively occurring in the embryonic loose mesenchymal
\end{abstract}

Correspondence to: Dr Lars Ährlund-Richter, Karolinska Institute, Department of Women's and Children's Health, Karolinska University Hospital, CCK R8:00, 17176 Stockholm, Sweden

E-mail: lars.ahrlund@ki.se

${ }^{*}$ Contributed equally

Key words: neuroblastoma, human, in vivo, model, stem cells stroma, resulting in a histology recapitulating NB native presentation in vivo. Whether this reflects the 'open' nature of loose mesenchyme more easily giving space to new cells compared to other more dense tissues, the rigidity of matrix providing physical cues modulating NB characteristics, or if embryonic loose mesenchyme may supply developmental cues that attracted or promoted the integration of NB, remains to be tested. We tentatively hypothesize that mature PSCT provide an embryonic niche well suited for in vivo studies on NB.

\section{Introduction}

Key knowledge is still missing for the successful cure of aggressive neuroblastoma (NB), representing one of the most deadly pediatric malignancies (1-4). Neuroblastoma is a small round cell tumor of childhood and is considered to arise from dedifferentiation of primordial neural crest cells that populate the sympathetic trunks and the adrenal medulla (reviewed in ref. 5). During this process, an aberrant response to microenvironment cues may play an important role in modulating the tumor phenotype, and hence also lead to the variable clinical presentations of NB in patients. The clinical presentation spans from a benign type with the ability to spontaneously regress to a variant with a high rate of recurrence, metastatic spread and a high frequency of therapy-resistance. Current consensus supports the importance of a strong interplay with the surrounding tissue promoting tumor growth and spread (6). Thus, pre-clinical studies of childhood NB would for increased relevance benefit from in vivo models better matching the embryonic neoplastic niche in early development from which this tumor is believed to originate.

Pluripotent stem cells (PSC) are defined by their ability to differentiate into any of the three germ layers and ectopic injections into mice repeatedly produce what is described as experimental teratoma $(7,8)$. Although generically referred to as a tumor, others and we have previously reported evidence that pluripotent stem cell-derived teratoma (PSCT) from PSC 
Table I. Overview of tumor cell lines and the experimental setup.

Number of injections and frequency of tumor takes

Tumor cell line

IMR-32

Kelly

Abdominal tumor mass

Brain metastasis

SK-N-BE(2)
Bone marrow metastasis
Genetic profile
MYCN amplification

$1 \mathrm{p}$ deletion

MYCN amplification

17q amplified

$11 q$ deletion

MYCN amplification

$1 \mathrm{p}$ deletion

Xenografts PSCT

$3 / 3$

$8 / 8$

$4 / 4$

$2 / 2$

$2 / 2$

$3 / 3$ with normal karyotype can be described as a failed embryonic process including increasingly chaotic embryonic tissues and an emerging organoid development (7,8-11). Despite lacking a developmental axis this process can show striking similarities to the events in the human embryo, including and often dominated by components of early neural development $(9,10)$. When compared to human embryos at diagnosed gestation stages, we observed minor kinetic deviations in the appearances of several organoid structures in PSCT developed from the well-studied human embryonic stem cell line HS181 (10). However, more advanced organoid structures are rare (7-10) and a prolonged immaturity of some neural components is a consistent finding also in benign PSCT induced by PSC with normal karyotype (reviewed in ref. 8). More specifically, such immature neural areas display a strong resemblance with primitive neuroectodermal tumors and a similar histology when appearing in patient samples is considered potentially malignant.

The stem cell induced teratoma environment has earlier been suggested as an experimental platform for studying human tumor cell lines of a variety of origins (ovarian, prostate, lung, glioblastoma, breast, colorectal cancer) (12-14). Similarly, we studied growth from a malignant melanoma cell line in human experimental teratoma and identified a de-differentiated tumor phenotype not present in xenografts from the same cell line (15), possibly indicating an adaptation of the tumor cells to the embryonic microenvironment.

To generate new insights and testing a principle of closer developmental match between the injected tumor and the PSCT microenvironment, we conducted experiments to explore the PSCT milieu specifically for in vivo support of tumors of embryonic origin. Proliferative tumor cells from the injections of three aggressive NB cell lines; IMR-32, Kelly and SK-N-BE(2) could readily be identified and growth was found exclusively integrated into areas of loose mesenchyme, presenting tumors with histological resemblance to clinical NB.

\section{Materials and methods}

Ethical permission. This study was performed in full accordance with permission for experiments using human embryonic stem cells, from the Local Ethics Committee at Karolinska Institute (114/00), and for animal experimentation from the regional ethics committee (Stockholm Northern Animal Review Board; Dnr S172-03 and N105/07).

Cell lines. The human embryonic stem cell lines HS181 (16) and H9 (17), both of 46:XX karyotype, were maintained in culture as previously described (18). The human male NB cell lines IMR-32, Kelly and SK-N-BE(2) were obtained from ATCC (Manassas, VA, USA). For genetic profile see Table I. All tumor cell lines were cultured in Iscove's modified Dulbecco's medium (IMDM) supplied with $10 \%$ fetal bovine serum (FBS) and 1\% penicillin/streptomycin (all from Invitrogen, Carlsbad, $\mathrm{CA}, \mathrm{USA}$ ), at $37^{\circ} \mathrm{C}, 6.8 \% \mathrm{CO}_{2}$, with high humidity.

Animals and generation of PSCT. SCID/Beige (C.B.-17/ GbmsTac-scid-bgDF N7; M\&B, Ry, Denmark), male mice, 6-8 weeks old, were used. PSCT were generated by injection of $10^{4}-10^{5} \mathrm{HS} 181$ under the testicular capsule of mice, as previously described $(8,9)$. PSCT were allowed to progress for 45 days before injected with NB tumor cells. The variations of germ layer formation in separate HS181 PSCT formed under the present conditions have been described previously in more detail and were in all cases minor $(9,15)$.

Injection of $N B$ cells. Cells from the indicated cell lines $\left(1 \times 10^{6}\right.$ cells in $20 \mu \mathrm{l}$ PBS), were injected into 45-day-old PSCT, or as a xenograft, directly under the testis capsule of mice anesthetized using isofluran (3\%). Injections of NB cells resulted in outgrowth in all cases. For number of injections and frequency of tumor takes see Table I. Animals were sacrificed two weeks after NB tumor cell injection, before reaching the limit of the accepted total size of the PSCT, including NB tumor growth, according to the ethical permit for the recipient animal.

Tissue preparation. Tumors were harvested after two weeks, fixed in $4 \%$ paraformaldehyde at $4^{\circ} \mathrm{C} 24$ hours and dehydrated through a graded series of alcohol to xylene, embedded in paraffin, then serially sectioned into $5-\mu$ m-thick sections and stained using standard hematoxylin and eosin (H\&E) staining for basic histological orientation. For each NB cell line, two xenografts and two PSCT with injected NB tumor were subjected to detailed screening of 10-30 sections each. 
Table II. Antibodies used.

\begin{tabular}{llll}
\hline Antigen & \multicolumn{1}{c}{ Source } & Dilution & \multicolumn{1}{c}{ 2nd detection } \\
\hline CD31 & DAKO, M 0823 & $1: 20$ & DakoCytomation EnVision+System-HRP \\
Chromogranin A & DAKO, A 0430 & $1: 2000$ & Bond Polymer Refine detection kit, Leica \\
Cripto-1 & Rockland, 600-401-99337 & $1: 400$ & 4+ Biotinylated Goat Anti-Rabbit-IgG, Biocare Medical \\
E-cadherin & Abcam, ab1416 & $1: 50$ & Vectastain Universal Elite ABC \\
HIF2 $\alpha$ & Novus Biologicals, NB100-132 & $1: 150$ & DakoCytomation EnVision+System-HRP \\
Ki67 & Abcam, ab833 & $1: 50$ & Vectastain Universal Elite ABC \\
Lefty & Santa Cruz, SC-7408 & $1: 25$ & 4+ Biotinylated Mouse Anti-Goat-IgG, Biocare Medical \\
Nodal & Life Span BioScience, LS-B3955 & $1: 100$ & 4+ Biotinylated Mouse Anti-Goat-IgG, Biocare Medical \\
Synaptophysin & Novocastra/Leica, NCL-L-Synap-299 & $1: 100$ & DakoCytomation EnVision+System-HRP \\
\hline
\end{tabular}

Table III. In vivo growth characteristics.

\begin{tabular}{lll}
\hline Tumor cell line & \multicolumn{1}{c}{ Xenograft (intra testis) } & \multicolumn{1}{c}{ PSCT } \\
\hline IMR-32 & $\begin{array}{l}\text { Poorly differentiated deliminated growth pattern } \\
\text { with a rich fibrovascular stroma. Polygonal } \\
\text { tumor cells with sheets of neurofibrillary material. } \\
\text { Poorly differentiated deliminated growth pattern } \\
\text { with a fine fibrovascular stroma. Moderate amount } \\
\text { of cytoplasm forming neuropil. }\end{array}$ & $\begin{array}{l}\text { Poorly differentiated, moderately small, } \\
\text { blastic cells with scant stroma. } \\
\text { No evident neuropil. }\end{array}$ \\
& $\begin{array}{l}\text { Undifferentiated stroma-poor appearance. } \\
\text { Small nuclei with condensed chromatin } \\
\text { and scant cytoplasm without any sign of } \\
\text { differentiation. Band-like necrotic areas }\end{array}$ \\
& $\begin{array}{l}\text { Undifferentiated solid areas as well as infiltrating } \\
\text { growth pattern. Pleomorphic nuclei and a }\end{array}$ & Similar to xenografts. \\
& moderate amount of eosinophilic cytoplasm &
\end{tabular}

Verification of NB tumor growth. Presence of NB tumor cells was verified using genetic identification as described previously (15). Human Y- and X-chromosomes was detected using mixed probes (CEP XY; Vysis Inc, Downers Grove, IL, USA) against the human X- and Y-chromosomes, thus positively separating human from mouse cells in xenografts, as well as separating IMR-32 cells (XX) from PSCT cells (XY), as previously described (15). For the identification of Kelly and SK-N-BE(2), both lacking stable Y-chromosome centromeres, the identification of their genetic MYCN amplification was performed using the probes LSI N-MYC, 2p24 (Vysis Inc).

Histological analysis. Areas of identified tumor growth were subjected to immunohistochemistry (IHC) using criterion for histological analysis of NB cell differentiation as those used in daily clinical practice and in other studies $(19,20)$.

Dilutions and conditions for the specific antibodies and secondary detection methods are presented in Table II. The IHC results were verified using positive control slides from tissues known to express the antigen of interest. Negative controls were performed by omitting primary antibody and by using non-specific antibodies as isotype controls in the same concentration as the primary antibody. Species-specificity of the human specific antibody for CD31 was verified internally in each staining, since both xenografts and the PSCT environment contain numerous vessels of murine origin, which were all negative.

Image analysis. Imaging was performed using a Zeiss Axiovert 200M microscope and Openlab 5.0 software. Images were adjusted for auto levels in Photoshop.

\section{Results}

Histopathology of NB cell lines in xenografts and PSCT. For all three NB cell lines the injections of $10^{6}$ cells yielded detectable tumor growth in all animals. The number of injections and frequency of tumor takes are summarized in Table I.

All NB cell lines presented growth in smaller nodules as well as larger areas of cohesive tumor growth. A consistent finding in both xenografts and PSCT was a poorly differentiated tumor with a variable amount of fibrovascular stroma, and no Schwann cell differentiation (Table III and illustrated in Fig. 1A-F for all three NB lines). Further, presence of a neuroendocrine phenotype as reflected by positive staining for synaptophysin and chromogranin A was a consistent finding for all three NB cell lines in both models, as exemplified by SK-N-BE(2) in Figs. 1E,F and 2D. 


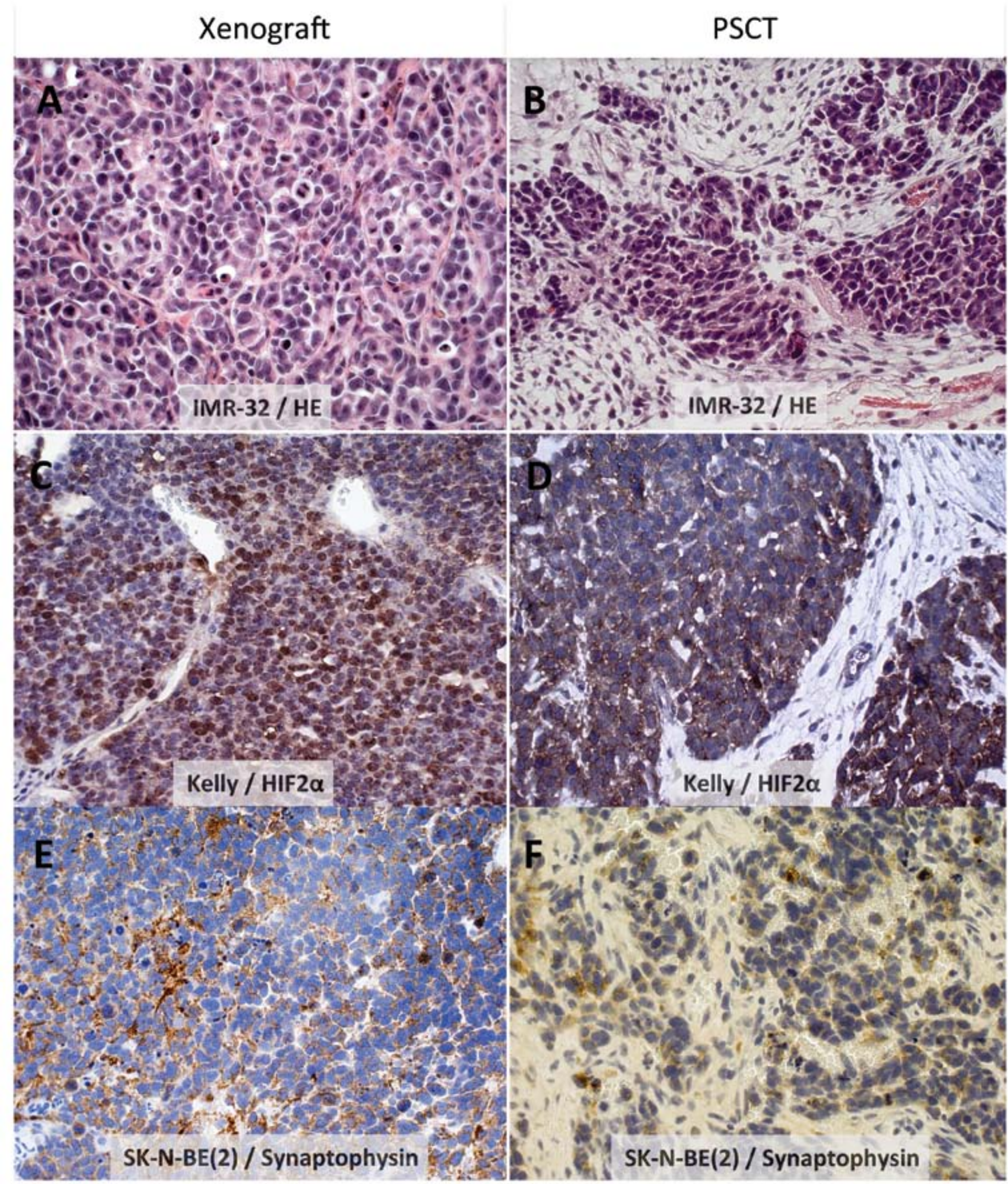

Figure 1. In vivo histology of IMR-32, Kelly and SK-N-BE(2). Section of PSCT two weeks after injection of NB cells, showing poorly differentiated tumors with a variable amount but mainly little fibrovascular stroma, and no Schwann cell differentiation. In the left hand panels (A, C and E) sections from intra testis xenografts, and in the right hand panels (B, D and F) sections after injections into PSCT. (A and B) IMR-32 cells stained for hematoxylin and eosin (H\&E). (C and D) Histology of Kelly tumor, stained for HIF2 $\alpha$. (E and F) Histology of SK-N-BE(2), stained for COX2. Original magnifications, x40.

The numbers of mitosis were similar in PSCT and xenografts; IMR-32 showed a proliferation index of $>70 \%$ in both models, while SK-N-BE and Kelly showed a proliferation index of $40-50 \%$.

Tropism of NB outgrowth in the PSCT microenvironment. For practical reasons the NB cells were injected into random positions in the PSCT cell mass. Small nodules of tumor growth were however detected exclusively in areas compatible with undifferentiated mesenchymal stroma, and never observed in areas with well differentiated somatic tissue i.e. bone, muscle, gut or areas of other easily identifiable tissue types.

Fig. 2A illustrates IMR-32 nodular growth (arrows) located in a loose mesenchyme environment, with the dotted boxed area illustrated at a higher magnification $(x 40)$ in Fig. 2B. Fig. 2C illustrates the nodular growth of Kelly, here located next to vessels staining for human CD31. Similarly, Fig. 2D illustrates small groups of SK-N-BE(2) tumor cells, here stained for Chromogranin A, embedded in loose mesenchyme.

Nodal-signaling pathway. Expression of Nodal and Cripto-1 but not Lefty was demonstrated for IMR-32 growth in xenografts (Fig. 3A-C) as well as in the PSCT microenvironment (Figs. 3D-F and 4A-C). Notably, in the PSCT environment, non-tumor areas of Cripto-1 positive condensing mesenchyme with similar histology as NB tumor growth could be shown to exhibit a reciprocal Nodal/Lefty expression pattern (compare Fig. 4A2 and A3 with B2 and B3). 


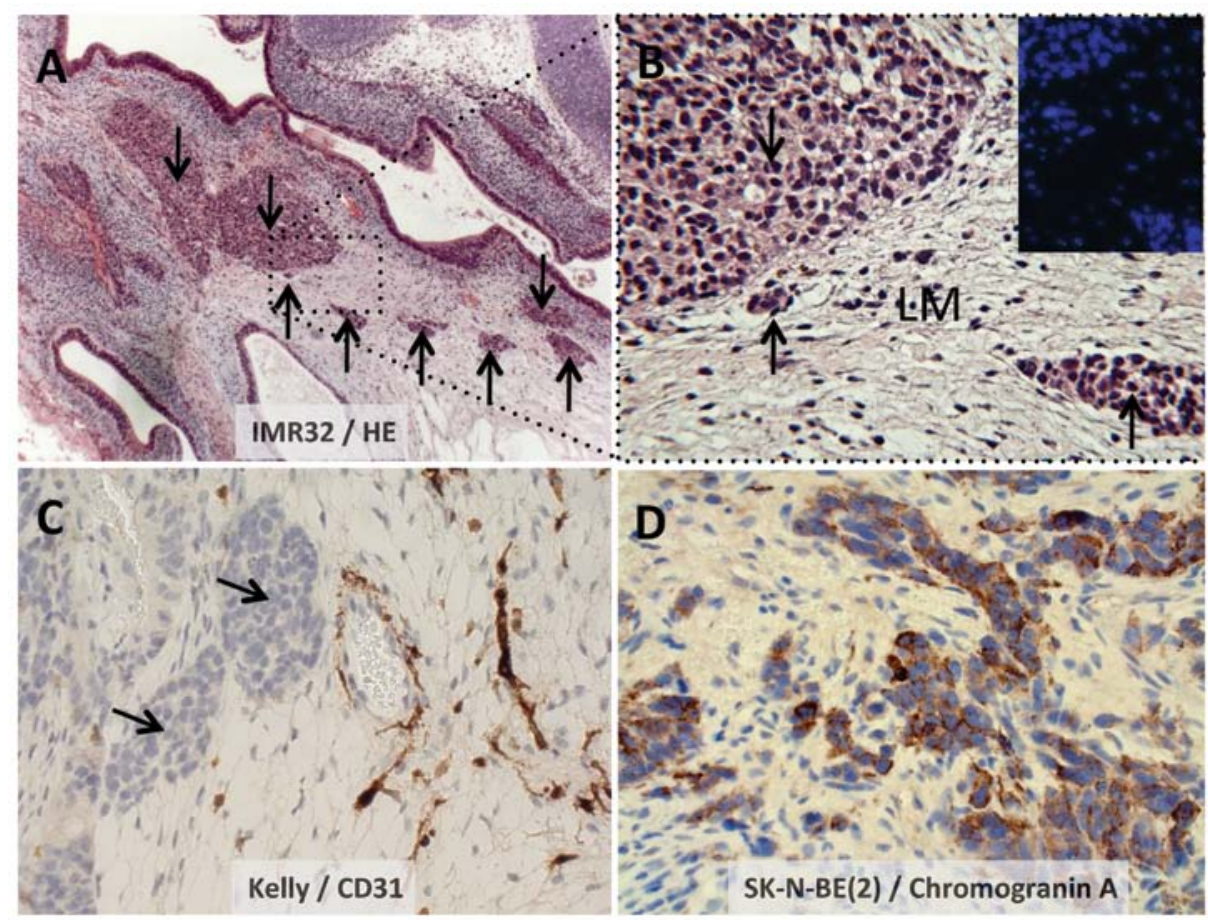

Figure 2. Nodular NB growth is in the PSCT located in embryonic loose mesenchyme. (A) Section of PSCT two weeks after injection with IMR-32, stained for hematoxylin and eosin (H\&E). Arrows indicate two separate areas with IMR-32 growth. (B) Higher magnification of boxed area. LM, loose mesenchyme. Inserted picture illustrates FISH analysis of adjacent section using human specific probes (green signal, human X-chromosome; red signal, human Y-chromosome) verifying location of IMR-23 cells. (C) Illustration of Kelly nodular growth (indicated by arrows) located in loose mesenchyme and close to vessels staining positive for human CD31. (D) Small groups of SK-N-BE(2) tumor cells staining positive for Chromogranin A, embedded in loose mesenchyme. Original magnifications, (A) $\mathrm{x} 10$; (B, C and D) $\mathrm{x} 40$.

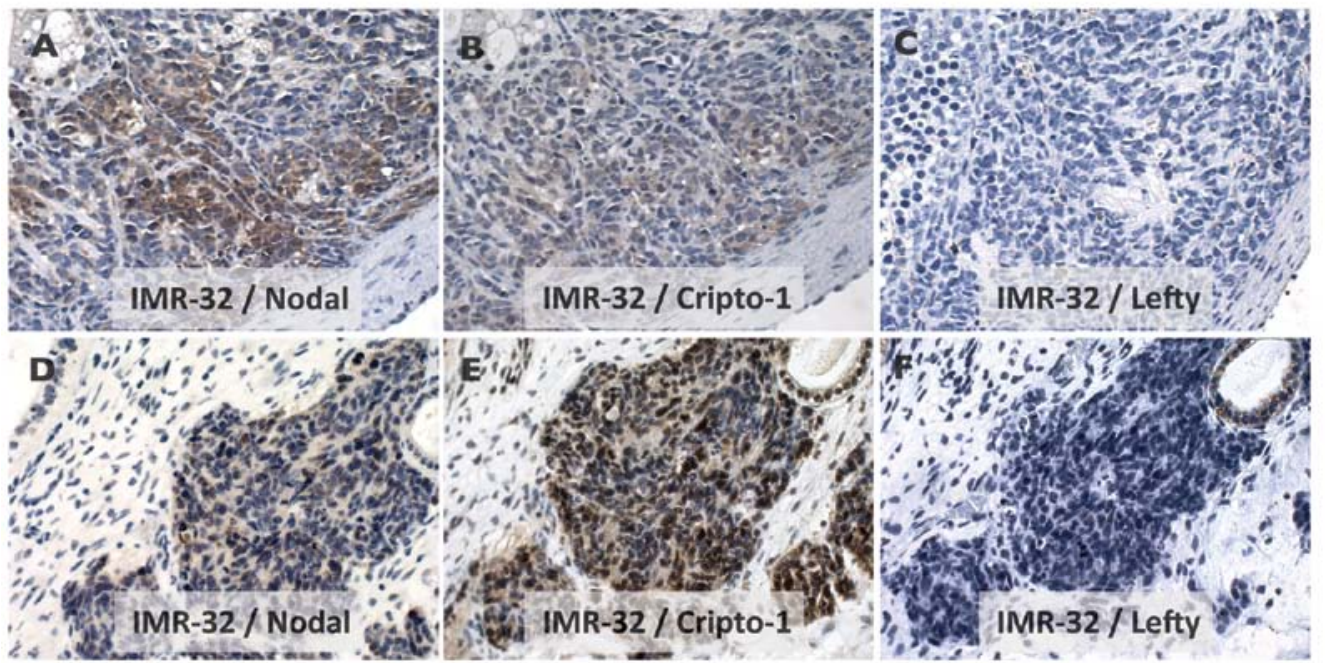

Figure 3. Expression of Nodal and Cripto but not Lefty in IMR-32 tumors. (A-C) Paraffin-embedded serial sections of IMR-32 xenografts, stained for (A) Nodal, (B) Cripto-1 or (C) Lefty. Seminiferous tubules are seen on the upper left side and the testis capsule in lower right corner. (D-F) Paraffin-embedded serial PSCT sections, showing an area of IMR-32 in the middle of the picture, stained positive for (D) Nodal, (E) Cripto-1 but not (F) Lefty. Original magnifications, x40.

\section{Discussion}

A comparable histology was observed for the three NB cell lines in the PSCT microenvironment or when growing as xenografts. However, the PSCT environment offered additional information and a conspicuous finding following injections into PSCT was that the smaller nodules of tumor growth, presumably repre- senting the initial manifestation of NB, showed for all three NB cell lines a strict tissue preference between the components of the embryonic process in the stem cell induced mature teratoma. Initial growth was observed exclusively in areas compatible with embryonic loose mesenchymal stroma. There was no evidence for NB cells occurring in areas compatible with bone, muscle, gut or areas of other identifiable tissue types. 

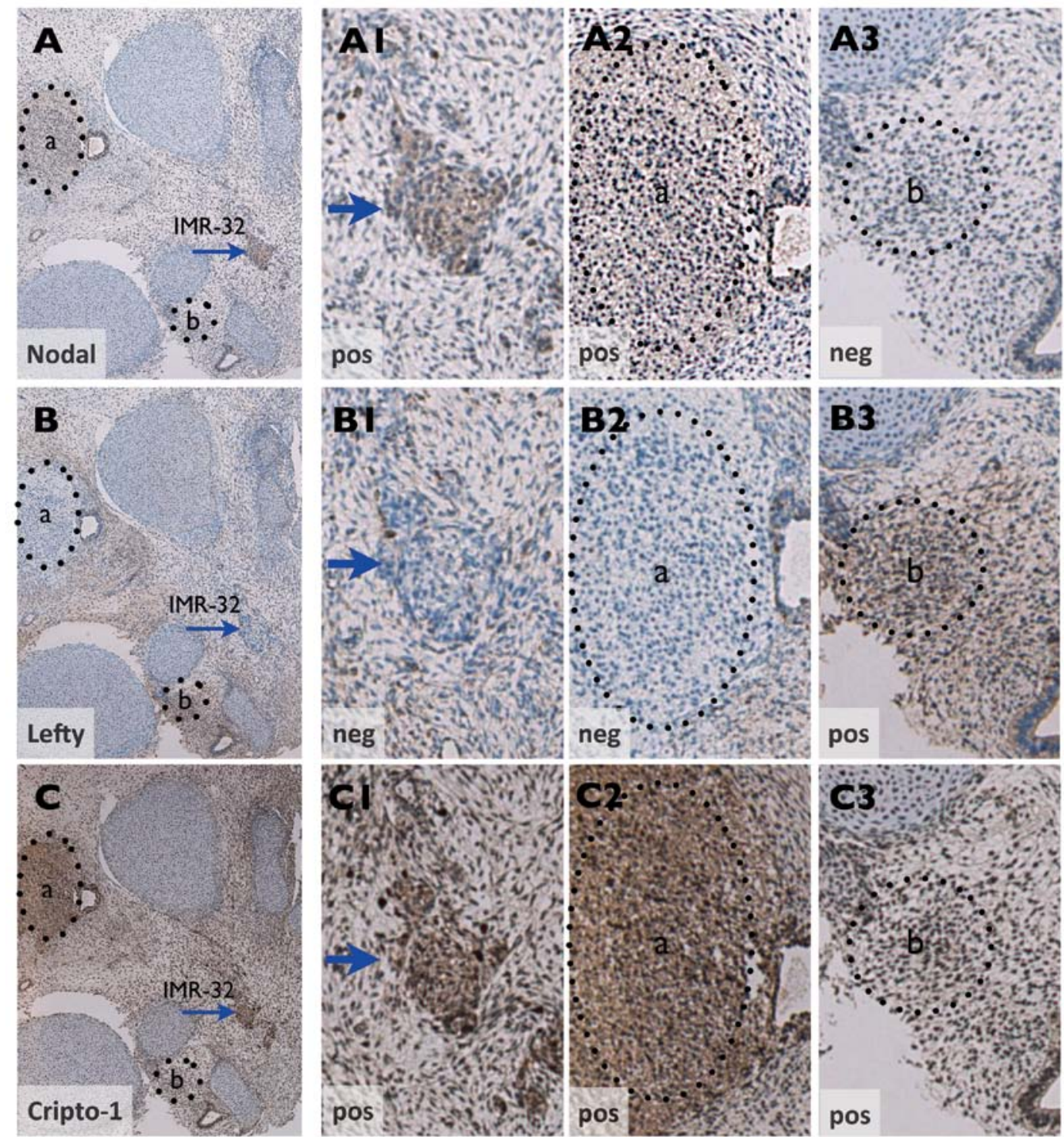

Figure 4. A reciprocal Nodal/Lefty expression of an IMR-32 tumor mirrors that of Cripto-1 positive condensing mesenchyme in PSCT. (A, B and C) Illustrates paraffin-embedded serial sections of PSCT two weeks after injection with IMR-32. Stained for (A) Nodal, (B) Lefty and (C) Cripto-1. Blue arrow indicates nodular growth of IMR-32 as verified by FISH analysis using human specific probes as described in text and Fig. 2 (data not shown). Two separate areas of condensing mesenchyme are indicated with dotted circles (a) and (b), respectively. (A1-3, B1-3 and C1-3) Show higher magnification of the staining in the indicated areas. Original magnifications (A, B and C) x5; (A1-3, B1-3 and C1-3) x20.

An upfront explanation regarding the mechanism behind this tropism could be the 'open' nature of the loose mesenchyme, i.e. more easily giving space to new cells compared to other more dense tissues. In this context, a recent report that rigidity of the extracellular matrix may provide a physical cue that can modulate NB differentiation, reduce NB proliferation and MYCN expression (21), is here of particular interest.

The ability of tumors to invade host tissues is dependent on signals mediated by several routes that normally enable important physiological functions, such as morphogenesis and angiogenesis. Notably, the mesenchyme of the early embryo is composed of a matrix of reticular fibers and myxoid ground substance and includes undifferentiated spindle or star shaped mesenchymal cells but also cells from other germ layers, e.g. ectodermal neural crest cells. The loose mesenchyme in PSCT is thus best understood as an embryonic tissue in which all connective tissue cell types may occur. The three main components of loose myxoid ground; glycosaminoglycans, proteoglycans and glycoproteins, are known components in cell-cell interactions and are here ideally situated for trophic factors. Thus, it can also be speculated from the presented findings that embryonic loose mesenchyme may supply developmental cues that attracted or promoted the integration of $\mathrm{NB}$ tumor cells in the present study.

Whether the explicit tropism identified for the three NB lines in the present study can be of a more general importance for $\mathrm{NB}$, or specific for the tumors used, remains to be determined using a larger panel of NB tumor lines.

Another typical finding was that the NB cells were regularly found in the immediate proximity of vessels. A homing to the perivascular niche is in line with the notion that $\mathrm{NB}$ could be considered a stem cell disease of the sympathetic 
nervous system (5) and that the perivascular niche supports stem cell self-renewal capacity and an undifferentiated state of neural tumor cells (22).

The cellular origin is not exactly known but NB is assumed to originate from neural crest precursor cells that specifically differentiate into the sympathoadrenal lineage. In this context, it is of interest that the timing of NB tumor cell injections used in this study, day 45 of the developing embryonic process in PSCT, overlaps with the presence in PSCT of neural components compatible with gestation stages immediately preceding the positioning of adrenal sympatical progenitors in embryonic mesenchyme (neural crest development; E25-35) $(3,19)$. Thus, we tentatively hypothesize that day 45 HS181 PSCT may provide a timely embryonic niche for in vivo studies on NB.

Some further indirect support for this hypothesis comes from the findings with the embryonic protein Nodal, member of the TGF- $\beta$ superfamily. Nodal acts as a morphogen in human early development, balanced by its antagonist Lefty, but is not expressed by most normal adult tissues (23-25). Dysregulated expression has however been detected in several malignancies and expression of Nodal has been linked with invasiveness and metastasis; as reported for melanoma, glioma, and cancers of breast endometrium and prostate (25-28). In a previous study using a melanoma cell line we detected Nodal positive tumor cells invading surrounding mesenchymal stroma in the PSCT model (15). We therefore wanted to know whether expression of Nodal could be similarly identified for NB. For this we selected IMR-32 based on its observed infiltrative growth into the surrounding stroma. Our results demonstrated that aggressive IMR-32 tumor growth indeed showed expression of both Nodal and its co-receptor Cripto-1, but not the antagonist Lefty. Intriguingly, the reciprocal Nodal/Lefty expression of IMR-32 in PSCT mirrored that of nearby Cripto-1 positive areas of condensing mesenchyme. This indicates that the expression of Nodal/Lefty in IMR-32 concurred with Nodal regulation in PSCT early embryonic patterning (24).

In conclusion, aiming for the principle of a closer developmental match between the tumor and the microenvironment this study demonstrates the feasibility of using the embryonic PSCT microenvironment for modeling in vivo growth of childhood neuroblastoma. The exclusive integration of NB cells into embryonic loose mesecnhymal stroma added histology with close recapitulation of NB native presentation in patients. This finding, together with the advantage of a species identity of surrounding stroma, suggests clear benefits for the PSCT model compared to xenotransplantation and the PSCT microenvironment to be an important well-needed complement for pre-clinical studies of NB, facilitating clinical translation. Our continued studies will involve expanding the NB model from cell lines to clinical tumor material from young NB patients. Considering the extremely poor prognosis of malevolent $\mathrm{NB}$, development of new clinically relevant models is of utmost importance.

\section{Acknowledgements}

This work was supported by the Swedish Childhood Cancer Foundation (08/092), the Cancer Research Funds of Radiumhemmet (09 1551 and 09 4271), Little Heroes Pediatric Cancer Research Foundation, Petrus and Augusta Hedlund's stiftelse and Karolinska Institutet. S. Jamil and R. Ali were supported by stipends from the Higher Education Commission of Pakistan.

\section{References}

1. Brodeur GM and Maris JM: Neuroblastoma. In: Principles and Practice of Pediatric Oncology. Pizzo PA and Poplack DG (eds). 5th edition. J.B. Lippincott \& Co, Philadelphia, PA, pp933-970, 2006.

2. Maris JM, Hogarty MD, Bagatell R and Cohn SL: Neuroblastoma. Lancet 369: 2106-2120, 2007.

3. Brodeur GM: Neuroblastoma: biological insights into a clinical enigma. Nat Rev Cancer 3: 203-216, 2003.

4. Johnsen JI, Kogner P, Albihn A and Henriksson MA: Embryonal neural tumours and cell death. Apoptosis 14: 424-438, 2009.

5. Mohlin SA, Wigerup C and Påhlman S: Neuroblastoma aggressiveness in relation to sympathetic neuronal differentiation stage. Semin Cancer Biol 21: 276-282, 2011.

6. Hanahan D and Weinberg RA: Hallmarks of cancer: the next generation. Cell 144: 646-674, 2011.

7. Blum B and Benvenisty N: The tumorigenicity of human embryonic stem cells. Adv Cancer Res 100: 133-158, 2008.

8. Cedervall J, Gertow K, Damjanov I and Ährlund-Richter L: Characterization of human pluripotent stem cell-derived teratoma. In: Human Stem Cell Manual. Loring JF and Peterson S (eds). 2nd edition. Elsevier Press, Philadelphia, PA, pp345-360, 2012.

9. Gertow K, Wolbank S, Rozell B, Sugars R, Andäng M, Parish CL, Imreh MP, Wendel M and Ährlund-Richter L: Organized development from human embryonic stem cells after injection into immunodeficient mice. Stem Cells Dev 13: 421-435, 2004.

10. Gertow K, Cedervall J, Jamil S, Ali R, Imreh MP, Parish CL, Imreh MP, Wendel M and Ährlund-Richter L: Early events in xenograft development from the human embryonic stem cell line HS181 - resemblance with an initial multiple epiblast formation. PLoS One 6: e27741, 2011.

11. Lensch, MW and Ince TA: The terminology of teratocarcinomas and teratomas. Nat Biotechnol 25: 1211, 2007.

12. Tzukerman M, Rosenberg T, Ravel Y, Reiter I, Coleman R and Skorecki K: An experimental platform for studying growth and invasiveness of tumor cells within teratomas derived from human embryonic stem cells. Proc Natl Acad Sci USA 100: $13507-13512,2003$.

13. Tzukerman M, Rosenberg $\mathrm{T}$, Reiter I, Ben-Eliezer $\mathrm{S}$, Denkberg G, Coleman R, Reiter Y and Skorecki K: The influence of a human embryonic stem cell-derived microenvironment on targeting of human solid tumor xenografts. Cancer Res 66: 3792-3801, 2006.

14. Katz E, Skorecki K and Tzukerman M: Niche-dependent tumorigenic capacity of malignant ovarian ascites-derived cancer cell subpopulations. Clin Cancer Res 15: 70-80, 2006.

15. Cedervall J, Jamil S, Prasmickaite L, Cheng Y, Eskandarpour M, Hansson J, Maelandsmo GM, Ringborg U, Gulyas M, Suo Z, Kanter L and Ährlund-Richter L: Species-specific in vivo engraftment of the human BL melanoma cell line results in an invasive dedifferentiated phenotype not present in xenografts. Cancer Res 69: 3746-3754, 2009.

16. Hovatta O, Mikkola M, Gertow K, Strömberg AM, Inzunza J, Hreinsson, J, Rozell B, Blennow E, Andäng $M$ and Ährlund-Richter L: A culture system using human foreskin fibroblasts as feeder cells allows production of human embryonic stem cells. Hum Reprod 18: 1404-1409, 2003.

17. Thomson JA, Itskovitz-Eldor J, Shapiro SS, Waknitz MA, Swiergiel JJ, Marshall VS and Jones JM: Embryonic stem cell lines derived from human blastocysts. Science 282: 1145-1147, 1998.

18. Imreh MP, Wolbank S, Unger C, Gertow K, Aints A, Szeles A, Imreh S, Hovatta O, Fried G, Dilber S and Ährlund-Richter L: Culture and expansion of the human embryonic stem cell line HS181, evaluated in a double-color system. Stem Cells Dev 13: 337-343, 2004.

19. Joshi VV, Cantor AB, Altshuler G, Larkin EW, Neill JS, Shuster JJ, Holbrook CT, Hayes FA and Castleberry RP: Recommendations for modification of terminology of neuroblastic tumor and prognostic significance of Shimada classification. A clinicopathologic study of 213 cases from the Pediatric Oncology Group. Cancer 69: 2183-2196, 1992. 
20. Shimada H, Umehara S, Monobe Y, Hachitanda Y, Nakagawa A, Goto S, Gerbing RB, Stram DO, Lukens JN and Matthay KK: International neuroblastoma pathology classification for prognostic evaluation of patients with peripheral neuroblastic tumors: a report from the Children's Cancer Group. Cancer 92: 2451-2461, 2001.

21. Lam WA, Cao L, Umesh V, Keung AJ, Sen S and Kumar S: Extracellular matrix rigidity modulates neuroblastoma cell differentiation and N-myc expression. Mol Cancer 9: 1-7, 2010.

22. Calabrese C, Poppleton H, Kocak M, Hogg TL, Fuller C, Hamner B, Oh EY, Gaber MW, Finklestein D, Allen M, Frank A, Bayazitov IT, Zakharenko SS, Gajjar A, Davidoff A and Gilbertson RJ: A perivascular niche for brain tumor stem cells. Cancer Cell 11: 69-82, 2007.

23. Schier AF: Nodal signaling in vertebrate development. Annu Rev Cell Dev Biol 19: 589-621, 2003.

24. Shen MM: Nodal signaling: developmental roles and regulation. Development 134: 1023-1034, 2007.
25. Postovit LM, Margaryan NV, Seftor EA, Kirschmann DA, Lipavsky A, Wheaton WW, Abbott DE, Seftor RE and Hendrix MJ: Human embryonic stem cell microenvironment suppresses the tumorigenic phenotype of aggressive cancer cells. Proc Natl Acad Sci USA 105: 4329-4334, 2008.

26. Papageorgiou I, Nicholls PK, Wang F, Lackmann M, Makanji Y, Salamonsen LA, Robertson DM and Harrison CA: Expression of nodal signalling components in cycling human endometrium and in endometrial cancer. Reprod Biol Endocrinol 7: $122,2009$.

27. Lee CC, Jan HJ, Lai JH, Ma HI, Hueng DY, Lee YC, Cheng YY, Liu LW, Wei HW and Lee HM: Nodal promotes growth and invasion in human gliomas. Oncogene 29: 3110-3123, 2010

28. Lawrence MG, Margaryan NV, Loessner D, Collins A, Kerr KM, Turner M, Seftor EA, Stephens CR, Lai J, APC BioResource, Postovit LM, Clements JA and Hendrix MJ: Reactivation of embryonic nodal signaling is associated with tumor progression and promotes the growth of prostate cancer cells. Prostate 71: 1198-1209, 2011. 